\title{
Ultra-High Resolution Imaging and Metrology with Low Vacuum SEM
}

\author{
B.L. Thiel, ${ }^{*}$ M. Toth, ${ }^{* *}$ and W.R. Knowles** \\ *College of Nanoscale Science and Engineering, University at Albany- SUNY, \\ 255 Fuller Road, Albany, NY 12203 \\ ** FEI Company, 1 Corporation Way \#2, Peabody, MA 01960
}

The ability to obtain high spatial resolution and high signal-to-noise ratio secondary electron (SE) images from multiphasic insulating specimens is essential for advancing many areas of nanotechnology, including critical dimension metrology of next generation microelectronics. Quality control for monitoring structures and processes at the nanometer scale places several requirements on an electron microscope: surface charging must be very well controlled and as uniform as possible; the working distance must be as short as possible to reduce the probe size; the beam energy must be as low as possible to maximize the surface sensitivity of the SE emissions; and lastly, the SE emissions must be amplified by a high gain, low noise process.

The new immersion lens based environmental SEM fulfills these requirements by providing an unprecedented combination of charge control and image resolution.[1] Gas amplification in these instruments is enhanced significantly through the confining effects of the magnetic field from the immersion lens. As an electron can only reach the detector anode after expending all of its kinetic energy through inelastic collisions with gas molecules, gas cascade amplification in this detector does not show the pressure sensitivity of Townsend gas capacitor style (i.e. linear electric field) detectors, and gives superior amplification at pressures below $200 \mathrm{~Pa}$. Gas gain with these detectors is also much less dependent on the distance from the sample surface to the detector. Accordingly, a very short working distance can be used, which capitalizes on the electron-optical advantages of the immersion lens. Furthermore, the combination of short working distance and low pressure allow very low beam energies to be used $(<1 \mathrm{keV})$, thus making the operating range comparable to those of high vacuum instruments. Finally, the electrode structure in these detectors has been designed such that the positive ion flux to the specimen surface will always provide a self-regulating surface potential near zero for all samples.

These instruments are ideal candidates for performing critical dimension metrology for microelectronics and other applications involving manufacturing at small lengthscales. Charging is often a major limiting factor, as it can cause feature distortion. Figure 1 shows a test structure for microelectronics fabrication of array of approximately $100 \mathrm{~nm}$ holes drilled into a silicon-nitride layer on a silicon substrate. If the structure is imaged under high vacuum at a fast scan rate (fig. 1a), charging is minimized, but the image is noisy. Slowing the scan rate improves the signal-to-noise ratio, but also results in charging artifacts (figs 1b-d). At the slowest scan rate (fig 1d), contrast from the holes inverts, and the hole pattern is distorted by beam deflection. Figure 2 shows the same test pattern imaged in low vacuum. At the fastest scan rate, noise is still a problem, but when the scan rate is slowed, image quality improves without image distortion or contrast 
inversion. Accurate information on the hole structure can then be obtained by analyzing line intensity profiles from the images as in Figure $3 a$ and $b$.

The electron-gas interactions and ion-surface interactions present in an ESEM introduce a number of factors which must be understood in order to interpret correctly. The influence of such processes -including gas amplification, skirt scattering and signal scavenging- on image formation will be discussed.

[1] B.L.Thiel, et al., Microscopy and Micoanalysis 10, Suppl. 2, 1058 (2004).

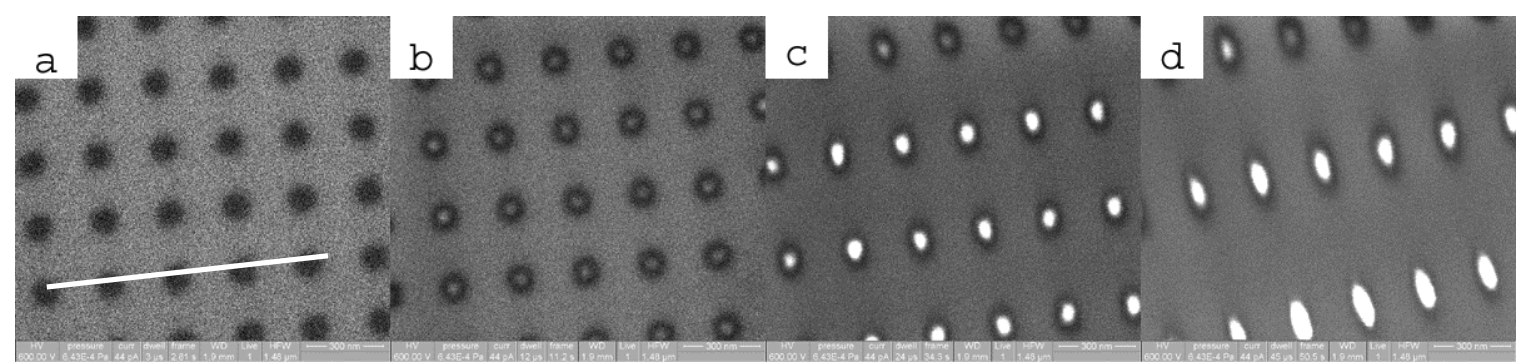

Figure 1. Test pattern of $100 \mathrm{~nm}$ holes in silicon nitride on a silicon substrate imaged in high vacuum. Dwell time per pixel (related to scan rate) is (a), $3 \mu \mathrm{s}$; (b) $12 \mu \mathrm{s}$; (c) $24 \mu \mathrm{s}$; (d) $45 \mu \mathrm{s}$. Beam energy $=600 \mathrm{eV}$, working distance $=1.9 \mathrm{~mm}$, beam current $44 \mathrm{pA}$.

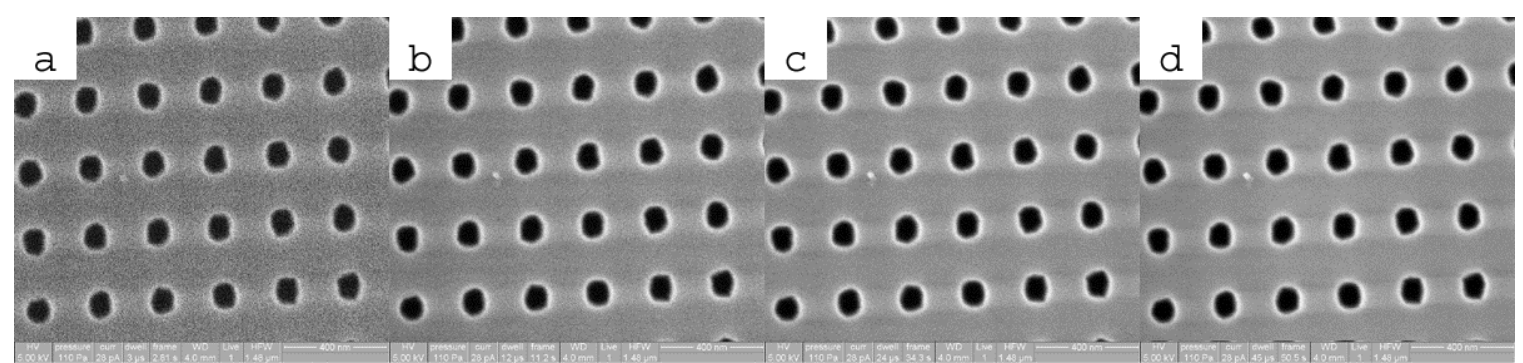

Figure 2. Test pattern from figure 1, imaged in low vacuum. Dwell times per pixel as in figure 1 . Beam energy $=5 \mathrm{keV}$, working distance $=4 \mathrm{~mm}$, beam current $28 \mathrm{pA}, 110 \mathrm{~Pa}$ water vapor.
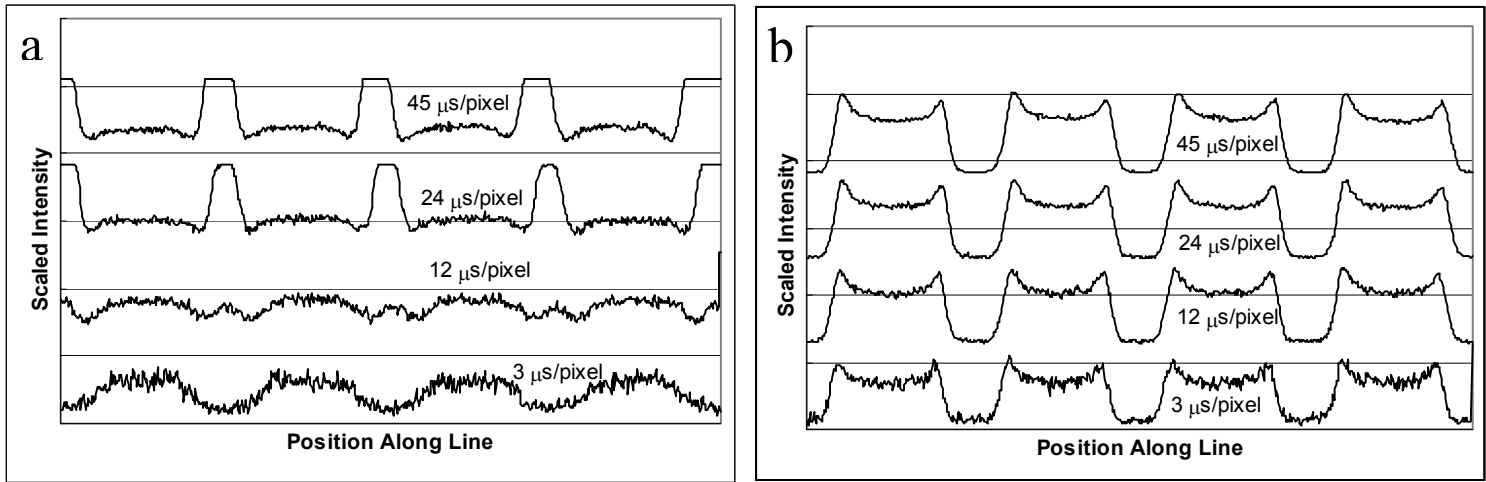

Figure 3. Line intensity profiles through three holes in image sets 1 and 2. The intensity inversion due to charging in the high vacuum case (a) is clear, as is the distortion of the spacing. Increasing the dwell time in low vacuum mode (b) improves the signal-to-noise ratio without causing inversion or distortion. 